\title{
Heterovalent ZnTe/GaSb and ZnSe/GaAs Grown by Molecular Beam Epitaxy
}

\author{
Brian D. Tracy ${ }^{1}$, David J. Smith ${ }^{1}$, Maxwell Lassise ${ }^{2}$ and Yong-Hang Zhang ${ }^{2}$ \\ 1. Department of Physics, Arizona State University, Tempe, AZ. \\ 2. School of Electrical, Computer and Energy Engineering, Arizona State University, Tempe, AZ.
}

The invention of semiconductor heterostructures and superlattices has enabled the discovery of novel low-dimensional phenomena such as quantum confinement, Bloch oscillations, and the fractional quantum Hall effect, in turn leading to technological breakthroughs in areas as diverse as light-emitting diodes (LEDs), quantum cascade lasers (QCLs), and quantum-well infrared photodetectors (QWIPs) [1]. Remarkably, all of these developments were based on structures having isovalent interfaces, such as IIVI/II-VI (e.g., CdTe/HgTe), III-V/III-V (e.g., GaAs/AlAs), or IV/IV (Si/Ge). However, the wide diversity of substrates that are commonly used makes it challenging to achieve monolithic device integration. Furthermore, lattice parameter mismatch at these interfaces invariably causes structural defects during epitaxial growth. These defects become a serious problem when attempting to optimize materials quality, while electron and hole traps associated with these defects are likely to have negative impact on electronic properties. One possible approach that could alleviate lattice mismatch issues is to combine II-VI and III-V compound semiconductors having the same zincblende-cubic crystal structure and similar lattice parameters. This study describes TEM characterization of several II-VI/III-V heterostructures grown by molecular beam epitaxy (MBE) with different growth parameters and structural designs. Cross-section samples suitable for TEM observation were prepared using standard mechanical polishing and argon-ion milling techniques, with the samples held at liquid-nitrogen temperature to minimize artefacts. All images were recorded with a Philips-FEI CM200 FEG TEM operated at $200 \mathrm{keV}$.

Figure 1 compares the microstructure of ZnTe films grown on GaSb (001) substrates using different initial growth conditions, and the insets show the corresponding high-resolution X-ray diffraction patterns. For the films shown in Figures 1a,b, respectively, there was a 10-second exposure of the GaSb surface to either $\mathrm{Zn}$ or Te flux before initiation of the ZnTe growth. The different wetting of the substrate surface has clearly had a remarkable effect on the subsequent crystal quality, possibly because of the formation of some deleterious interfacial compound [2]. Figure 2 compares three $\mathrm{ZnTe} / \mathrm{InAsSb} / \mathrm{ZnTe}$ quantum wells grown on $\mathrm{GaSb}$ with different $\mathrm{Zn}$ :Te partial pressures as indicated. The morphology of the ZnTe thin films does not seem to be much affected by this growth parameter over the range studied. However, the InAsSb films are non-uniform in thickness, which is attributed to the lower than optimum growth temperature that is required to protect the II/VI film, and also prevent poisoning of the III/V chamber by desorption of any II/VI material during the III/V growth. Figure 3 shows low magnification and high magnification images of a $\mathrm{ZnSe} / \mathrm{GaAs}$ quantum well. The GaAs quantum well appears much flatter compared with the InAsSb despite a similar temperature difference. However, the $\mathrm{ZnSe}$ films for most of the growth experiments displayed considerable amounts of stacking faults, as shown by the examples in Figure 3a,b [3].

\section{References:}

[1] Z.I. Alferov, Semiconductors 32 (1998) p. 1.

[2] M.P. Halsall et al, Appl. Phys. Lett. 60 (1992) p. 2129.

[3] The authors acknowledge support from AFOSR and the use of facilities within the John M. Cowley Center for High Resolution Electron Microscopy at Arizona State University. 


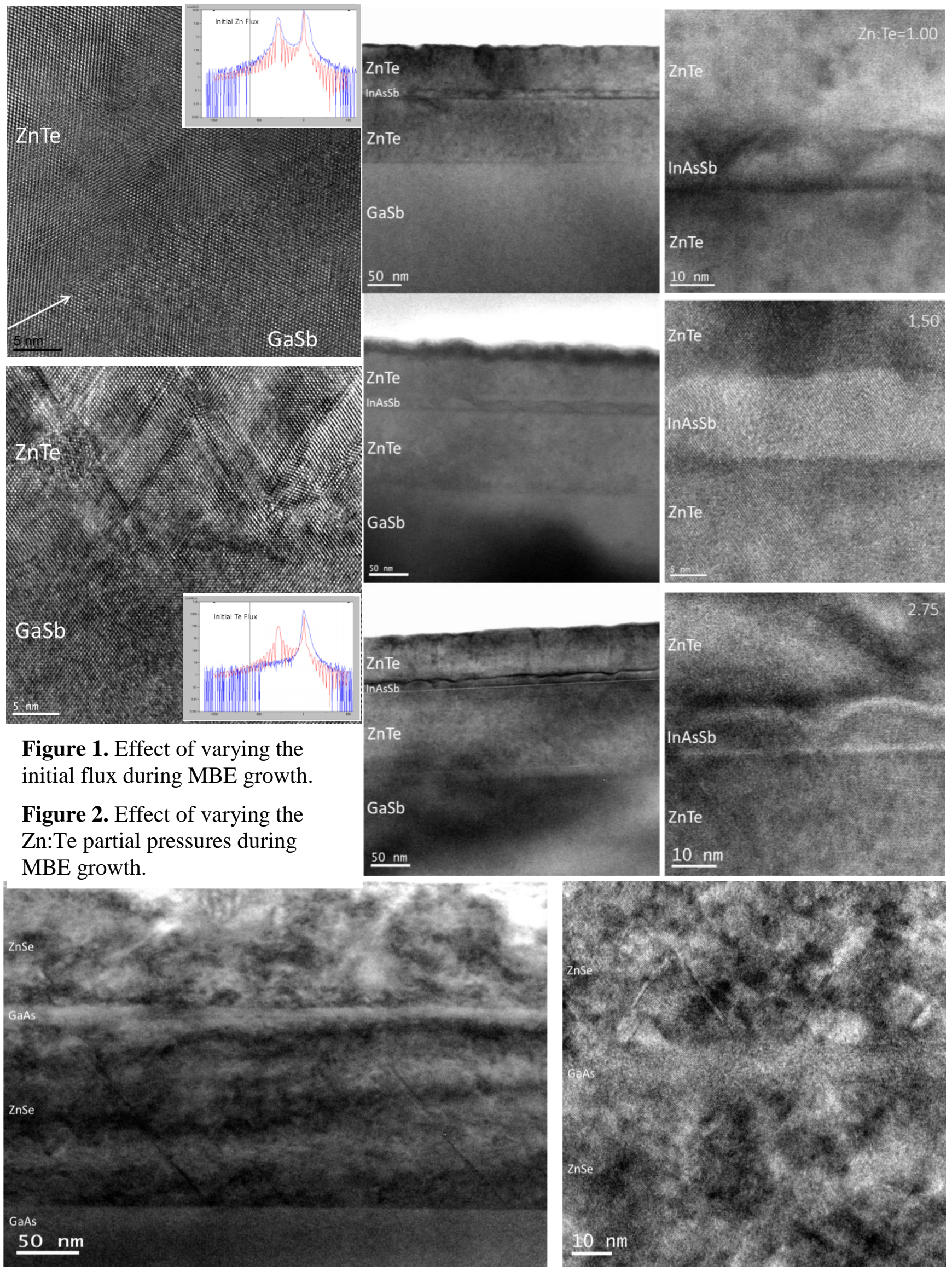

Figure 3. TEM images showing stacking faults primarily originating at $\mathrm{ZnSe} / \mathrm{GaAs}$ interfaces. 